\title{
The Dirichlet problem for the prescribed Ricci curvature equation on cohomogeneity one manifolds
}

\author{
Artem Pulemotov ${ }^{1,2}$
}

Received: 3 February 2015 / Accepted: 17 June 2015 / Published online: 27 June 2015

(C) Fondazione Annali di Matematica Pura ed Applicata and Springer-Verlag Berlin Heidelberg 2015

\begin{abstract}
Let $M$ be a domain enclosed between two principal orbits on a cohomogeneity one manifold $M_{1}$. Suppose that $T$ and $R$ are symmetric invariant $(0,2)$-tensor fields on $M$ and $\partial M$, respectively. The paper studies the prescribed Ricci curvature equation $\operatorname{Ric}(G)=T$ for a Riemannian metric $G$ on $M$ subject to the boundary condition $G_{\partial M}=R$ (the notation $G_{\partial M}$ here stands for the metric induced by $G$ on $\partial M$ ). Imposing a standard assumption on $M_{1}$, we describe a set of requirements on $T$ and $R$ that guarantee global and local solvability.
\end{abstract}

Keywords Ricci curvature $\cdot$ Dirichlet problem · Cohomogeneity one

Mathematics Subject Classification 53B20 $\cdot 53 \mathrm{C} 20 \cdot 58 \mathrm{~J} 32$

\section{Introduction}

Suppose that $M$ is a smooth manifold of dimension 3 or higher (possibly with boundary) and $T$ is a symmetric $(0,2)$-tensor field on $M$. The present paper investigates the prescribed Ricci curvature equation

$$
\operatorname{Ric}(G)=T,
$$

where the unknown $G$ is a Riemannian metric on $M$. Mathematicians have been studying (1.1) since at least the early 1980s. We invite the reader to see [4,7] for the history of the subject. The list of recent references not mentioned in [4,7] includes but is not limited to [15, 16,22,26-28].

The author is the recipient of an Australian Research Council Discovery Early-Career Researcher Award DE150101548.

$凶$ Artem Pulemotov

a.pulemotov@uq.edu.au

1 School of Mathematics and Physics, The University of Queensland, St Lucia, QLD 4072, Australia

2 Department of Mathematics, The University of Chicago, 5734 South University Ave, Chicago, IL 60637-1514, USA 
The solvability of boundary-value problems for Eq. (1.1) is, by and large, an unexplored topic. The author of the present paper made progress on this topic in [24]. The main theorems of [24] concern the local solvability of Dirichlet- and Neumann-type problems for (1.1) (i.e. solvability in a neighbourhood of a boundary point on $M$ ).

It is worth noting that D. DeTurck's study of (1.1) underlay his discovery of the DeTurck trick. In a similar fashion, new knowledge about boundary-value problems for (1.1) may help answer questions about the existence and uniqueness of solutions to boundary-value problems for the Ricci flow and the Einstein equation. Such questions were investigated in $[1,2,5,10,17,23,25,29]$ and other works. A large number still remain open.

Let $M_{1}$ be a smooth connected manifold of dimension 3 or higher with $\partial M_{1}=\emptyset$. Consider a compact Lie group $\mathcal{G}$ acting on $M_{1}$. Suppose that the orbit space $M_{1} / \mathcal{G}$ is one dimensional. It is then customary to call $M_{1}$ a cohomogeneity one manifold. Such manifolds enjoy numerous applications in geometry and mathematical physics; see, e.g. [6,8,11,13] and the references of [21]. In what follows, we suppose that $M$ is the closure of a domain on $M_{1}$ contained between two principal $\mathcal{G}$-orbits. The boundary of $M$ is then equal to the union of these orbits. It will be convenient for us to assume that the tensor field $T$ introduced above is defined on all of $M_{1}$, not just $M$. The purpose of the present paper is to study the global and local existence of solutions to a Dirichlet-type problem for Eq. (1.1) on $M$. Our main results are stated as Theorems 2.2, 2.5, and 2.6.

\section{Main results}

Because the space $M_{1} / \mathcal{G}$ is one dimensional, it must be homeomorphic to the real line, the closed interval, the half-line, or the circle. In the first and the fourth case, there are no singular orbits on $M_{1}$. For the sake of convenience, we will assume that $M_{1} / \mathcal{G}$ is homeomorphic to $\mathbb{R}$. It is easy to state analogues of our theorems in the situations where this assumption does not hold. Pick a point in $M_{1}$ and denote by $\mathcal{H}$ the isotropy group of this point. We will use the symbol $\mathfrak{g}$ for the Lie algebra of $\mathcal{G}$. Choose an $\operatorname{Ad}(\mathcal{G})$-invariant scalar product $Q$ on $\mathfrak{g}$. Suppose that $\mathfrak{p}$ is the orthogonal complement of the Lie algebra of $\mathcal{H}$ in $\mathfrak{g}$ with respect to $Q$. We standardly identify $\mathfrak{p}$ with the tangent space of $\mathcal{G} / \mathcal{H}$ at $\mathcal{H}$. The isotropy representation of $\mathcal{G} / \mathcal{H}$ then yields the structure of an $\mathcal{H}$-module on $\mathfrak{p}$. The following requirement will be imposed throughout the rest of the paper.

Hypothesis 2.1 The $\mathcal{H}$-module $\mathfrak{p}$ appears as an orthogonal sum

$$
\mathfrak{p}=\mathfrak{p}_{1} \oplus \cdots \oplus \mathfrak{p}_{n}
$$

of pairwise nonisomorphic irreducible $\mathcal{H}$-modules $\mathfrak{p}_{1}, \ldots, \mathfrak{p}_{n}$.

Roughly speaking, Hypothesis 2.1 ensures that $\mathcal{G}$-invariant $(0,2)$-tensor fields on $\mathcal{G} / \mathcal{H}$ are diagonal; cf. (2.4) and (2.6) below. This hypothesis is rather standard. It has come up in several papers including $[12,13]$.

Let the tensor field $T$ be $\mathcal{G}$-invariant. Assume that it is possible to construct a diffeomorphism

$$
\Psi: \mathbb{R} \times(\mathcal{G} / \mathcal{H}) \rightarrow M_{1}
$$

such that the map $\Psi(r, \cdot)$ is $\mathcal{G}$-equivariant for every $r \in \mathbb{R}$ and the equality

$$
\Psi^{*} T=\mathrm{d} r \otimes \mathrm{d} r+T_{r}, \quad r \in \mathbb{R},
$$


holds true. Here, $T_{r}$ is a $(0,2)$-tensor field on $\mathcal{G} / \mathcal{H}$ defined for each $r \in \mathbb{R}$. It is fully determined by how it acts on $\mathfrak{p}$. In view of Hypothesis 2.1, there exist smooth functions $\phi_{1}, \ldots, \phi_{n}$ from $\mathbb{R}$ to $\mathbb{R}$ such that

$$
T_{r}(X, Y)=\phi_{1}(r) Q\left(\operatorname{pr}_{\mathfrak{p}_{1}} X, \operatorname{pr}_{\mathfrak{p}_{1}} Y\right)+\cdots+\phi_{n}(r) Q\left(\operatorname{pr}_{\mathfrak{p}_{n}} X, \operatorname{pr}_{\mathfrak{p}_{n}} Y\right), \quad X, Y \in \mathfrak{p} .
$$

The notation $\operatorname{pr}_{\mathfrak{p}_{k}} X$ and $\operatorname{pr}_{\mathfrak{p}_{k}} Y$ refers to the projections of $X$ and $Y$ onto $\mathfrak{p}_{k}$ for $k=1, \ldots, n$.

If the tensor field $T$ is positive definite, it is always possible to construct the diffeomorphism $\Psi$. Indeed, in this case, we can interpret $T$ as a Riemannian metric on $M_{1}$ and consider a unit speed geodesic $\eta$ with respect to this metric. Assuming $\eta$ is orthogonal to all the principal orbits, we define $\Psi(r, g \mathcal{H})=g \eta(r)$ for all $r \in \mathbb{R}$ and $g \in \mathcal{G}$. This construction is quite standard; for example, it was used in $[13,18,21]$.

In what follows, we suppose that $\Psi$ is the identity map and

$$
M=[0, \sigma] \times \mathcal{G} / \mathcal{H}
$$

for some $\sigma>0$. This does not cause any loss of generality. Let $R$ be a symmetric positive definite $\mathcal{G}$-invariant $(0,2)$-tensor field on $\partial M$. We will use $R$ to impose a boundary condition on (1.1). Denote by $R^{0}$ and $R^{\sigma}$ the restrictions of $R$ to $\{0\} \times \mathcal{G} / \mathcal{H}$ and $\{\sigma\} \times \mathcal{G} / \mathcal{H}$, respectively. Given $\tau \in[0, \sigma]$, it will be convenient for us to identify the tangent spaces to $\{\tau\} \times \mathcal{G} / \mathcal{H}$ at the point $\{\tau\} \times \mathcal{H}$ with $\mathfrak{p}$ in the natural way. We observe that $R$ is fully determined by how $R^{0}$ and $R^{\sigma}$ act on $\mathfrak{p}$. Thanks to Hypothesis 2.1 , there exist positive numbers $a_{1}, \ldots, a_{n}$ and $b_{1}, \ldots, b_{n}$ satisfying the equalities

$$
\begin{aligned}
& R^{0}(X, Y)=a_{1}^{2} Q\left(\operatorname{pr}_{\mathfrak{p}_{1}} X, \operatorname{pr}_{\mathfrak{p}_{1}} Y\right)+\cdots+a_{n}^{2} Q\left(\operatorname{pr}_{\mathfrak{p}_{n}} X, \operatorname{pr}_{\mathfrak{p}_{n}} Y\right), \\
& R^{\sigma}(X, Y)=b_{1}^{2} Q\left(\operatorname{pr}_{\mathfrak{p}_{1}} X, \operatorname{pr}_{\mathfrak{p}_{1}} Y\right)+\cdots+b_{n}^{2} Q\left(\operatorname{pr}_{\mathfrak{p}_{n}} X, \operatorname{pr}_{\mathfrak{p}_{n}} Y\right), \quad X, Y \in \mathfrak{p} .
\end{aligned}
$$

Fix a number $\alpha>0$ such that

$$
\left|\phi_{i}(r)\right| \leq \alpha, \quad i=1, \ldots, n, \quad r \in[0, \sigma],
$$

along with a pair of numbers $\omega_{1}, \omega_{2}>0$ such that

$$
\omega_{1} \leq a_{i}, b_{i} \leq \omega_{2}, \quad i=1, \ldots, n .
$$

Denote by $d_{i}$ the dimension of $\mathfrak{p}_{i}$ for $i=1, \ldots, n$. Given a Riemannian metric $G$ defined on a neighbourhood of $\partial M$, we write $G_{\partial M}$ for the metric induced by $G$ on $\partial M$. Our first result is a sufficient condition for the global solvability of a Dirichlet-type problem for (1.1).

Theorem 2.2 There exist functions $\rho_{0}:(0, \infty)^{2} \rightarrow(0, \infty)$ and $\sigma_{0}:(0, \infty)^{5} \rightarrow(0, \infty)$, both independent of the tensor fields $T$ and $R$, such that the following statement is satisfied: if the formulas

$$
\left|\frac{\mathrm{d}}{\mathrm{d} r} \phi_{i}(r)\right| \leq c_{1} \sigma, \quad\left|a_{i}-b_{i}\right| \leq c_{2} \sigma^{2}, \quad i=1, \ldots, n, r \in[0, \sigma],
$$

and the formulas

$$
\begin{aligned}
& \sum_{i=1}^{n} d_{i}\left(\max \left\{\phi_{i}(r), 0\right\}+\frac{\omega_{2}^{2}}{\omega_{1}^{2}} \min \left\{\phi_{i}(r), 0\right\}\right)>\rho_{0}\left(\omega_{1}, \omega_{2}\right), \\
& \sigma<\sigma_{0}\left(\alpha, \omega_{1}, \omega_{2}, c_{1}, c_{2}\right), \quad r \in[0, \sigma],
\end{aligned}
$$

hold for some $c_{1}, c_{2}>0$, then the manifold $M$ supports a $\mathcal{G}$-invariant Riemannian metric $G$ solving the equation $\operatorname{Ric}(G)=T$ on $M$ under the boundary condition $G_{\partial M}=R$. 
Remark 2.3 When proving Theorem 2.2, we will obtain explicit expressions for $\rho_{0}$ and $\sigma_{0}$. These expressions (at least the one for $\sigma_{0}$ ) will be rather unsightly.

Remark 2.4 The first formula in (2.9) essentially forbids the part of $T$ tangent to the $\mathcal{G}$-orbits to change dramatically from one orbit to another. The second one says that $R^{0}$ should not be very different from $R^{\sigma}$. Note that formulas (2.9) are automatically satisfied when $R^{0}$ coincides with $R^{\sigma}$ on $\mathfrak{p}$ and $\phi_{1}, \ldots, \phi_{n}$ are constant. The meaning of (2.10) is that the tensor field $T$ has to be large in the directions tangent to the $\mathcal{G}$-orbits and small in the direction transverse to the $\mathcal{G}$-orbits.

Our second result establishes the local solvability of (1.1) in the interior of $M$. Given $\tau \in[0, \sigma]$, we denote by $\Gamma^{\tau}$ the $\mathcal{G}$-orbit $\{\tau\} \times \mathcal{G} / \mathcal{H}$ on $M$.

Theorem 2.5 For each $\tau \in(0, \sigma)$, there exists a $\mathcal{G}$-invariant Riemannian metric $G^{\tau}$ on $M$ such that the equality $\operatorname{Ric}\left(G^{\tau}\right)=T$ holds on some neighbourhood of $\Gamma^{\tau}$.

Next, we establish the local solvability of (1.1) near $\partial M$.

Theorem 2.6 There exists a $\mathcal{G}$-invariant Riemannian metric $G^{\text {bdy }}$ on $M$ such that $\operatorname{Ric}\left(G^{b d y}\right)=T$ on some neighbourhood of $\partial M$ and $G_{\partial M}^{b d y}=R$.

The proofs of Theorems 2.5 and 2.6 will rely on Proposition 3.9 appearing below. This proposition will also demonstrate that a $\mathcal{G}$-invariant metric solving the prescribed Ricci curvature equation near a $\mathcal{G}$-orbit on $M$ is uniquely determined by the metric it induces on this $\mathcal{G}$-orbit and by the orbit's second fundamental form.

Remark 2.7 Assume that $\mathcal{G}$ is the special orthogonal group $\mathrm{SO}(d)$ and $M_{1}$ coincides with $\mathbb{R}^{d}$ less a closed ball around the origin. One may then be able to study boundary-value problems for (1.1) with the methods of [9]; see also [19]. These methods consist in reducing the prescribed Ricci curvature equation to a first-order ordinary differential equation for a single real-value function. The authors of [9] were able to achieve such a reduction by exploiting the fact that $\mathrm{SO}(d)$-invariant metrics on $\mathbb{R}^{d}$ are globally conformally flat. In essence, their arguments relied on a clever change of variable in the prescribed Ricci curvature equation.

Remark 2.8 Instead of requiring that Hypothesis 2.1 hold for $\mathcal{G} / \mathcal{H}$, we may assume that $\mathcal{G} / \mathcal{H}$ is an abelian Lie group. The $\mathcal{H}$-module $\mathfrak{p}$ can then be written in the form (2.1) with the $\mathcal{H}$-modules $\mathfrak{p}_{k}$ being one dimensional for all $k=1, \ldots, n$. As before, we suppose that there exists a diffeomorphism $\Psi$ satisfying formula (2.3). In our current situation, however, it is not necessarily the case that there are smooth functions $\phi_{1}, \ldots, \phi_{n}$ from $[0, \sigma]$ to $\mathbb{R}$ obeying equality (2.4). Assume that such functions do exist. Suppose also that one can find positive numbers $a_{1}, \ldots, a_{n}$ and $b_{1}, \ldots, b_{n}$ such that (2.6) holds. Thus, we demand that $T$ and $R$ be diagonal with respect to (2.1). It is then possible to prove the assertions of Theorems 2.5 , 2.6, and 2.2 using the reasoning of Sect. 3.

Remark 2.9 Instead of assuming the existence of $\Psi$ above, one may assume that there is a diffeomorphism such that (2.3) holds with this diffeomorphism substituted for $\Psi$ and a minus sign in front of $\mathrm{d} r \otimes \mathrm{d} r$. The techniques in the present paper seem to be effective for treating this case. We will not dwell on any further details.

Example 2.10 Given an interval $I \subset \mathbb{R}$ and a number $\epsilon \geq 0$, denote

$$
\mathcal{D}_{I}=\left\{(x, y) \in \mathbb{R}^{2} \mid \sqrt{x^{2}+y^{2}} \in I\right\}, \quad \mathcal{S}_{\epsilon}=\left\{(x, y) \in \mathbb{R}^{2} \mid x^{2}+y^{2}=\epsilon^{2}\right\} .
$$


Assume that $\mathcal{G}$ is the product $\mathrm{SO}(2) \times \mathrm{SO}(2)$. Define $M_{1}$ to equal $\mathcal{D}_{\left(\frac{\chi}{2}, 2\right)} \times \mathcal{S}_{1}$ with $\chi>0$. The standard action of $\mathrm{SO}(2)$ on $\mathbb{R}^{2}$ gives rise to an action of $\mathcal{G}$ on $M_{1}$. The orbits of this action are the tori $\mathcal{S}_{\epsilon} \times \mathcal{S}_{1}$ with $\epsilon \in\left(\frac{\chi}{2}, 2\right)$. The isotropy group of an arbitrarily chosen point in $M_{1}$ consists of nothing but the identity element in $\mathcal{G}$. Consider a $(0,2)$-tensor field $T$ on $M_{1}$. It is convenient for us to assume that $T$ is positive definite, although this assumption can be relaxed. Suppose that $T$ is rotationally symmetric in the sense of [14,24]. This means $T$ is $\mathcal{G}$-invariant and diagonal with respect to the cylindrical coordinates on $\mathcal{D}_{[0,2]} \times \mathcal{S}_{1}$. We define $M$ to equal $\mathcal{D}_{[\chi, 1]} \times \mathcal{S}_{1}$. Thus, $M$ is a solid torus less a neighbourhood of the core circle. Consider a symmetric positive definite $(0,2)$-tensor field $R$ on $\partial M$. We suppose that $R$ is $\mathcal{G}$-invariant and diagonal in the coordinates induced on $\partial M$ by the cylindrical coordinates on $\mathcal{D}_{[0,2]} \times \mathcal{S}_{1}$. In the current setting, Theorem 2.2 (along with Remark 2.8) yields a sufficient condition for the solvability of the equation $\operatorname{Ric}(G)=T$ on all of $M$, subject to $G_{\partial M}=R$. No such condition previously appeared in the literature. Theorems 2.5 and 2.6 imply local solvability; cf. [24].

\section{The proofs}

In what follows, we assume that $T$ is positive definite and $c_{1}=c_{2}=1$. Thus, the function $\sigma_{0}$, whose existence Theorem 2.2 asserts, becomes a function of three variables, not five. These assumptions will make our arguments easier to follow. Removing them is straightforward.

\subsection{Preparatory material}

We begin by stating a formula for the Ricci curvature of a $\mathcal{G}$-invariant metric on $M$. This formula will involve two arrays of numbers, $\left(\beta_{k}\right)_{k=1}^{n}$ and $\left(\gamma_{k, l}^{m}\right)_{k, l, m=1}^{n}$. In order to introduce them, denote by $[\cdot, \cdot]$ and $P$ the Lie bracket and the Killing form of the Lie algebra $\mathfrak{g}$. The irreducibility of the summands in decomposition (2.1) implies the existence of nonnegative numbers $\beta_{1}, \ldots, \beta_{n}$ such that

$$
P(X, Y)=-\beta_{k} Q(X, Y), \quad k=1, \ldots, n, X, Y \in \mathfrak{p}_{k} .
$$

Because the group $\mathcal{G}$ is compact and Hypothesis 2.1 holds, at least one of these numbers must be strictly positive. Suppose that $d$ is the dimension of $M$. We choose a $Q$-orthonormal basis $\left(\tilde{e}_{i}\right)_{i=1}^{d-1}$ of the space $\mathfrak{p}$ adapted to (2.1). In addition to $\beta_{1}, \ldots, \beta_{n}$, let us define

$$
\gamma_{k, l}^{m}=\frac{1}{d_{k}} \sum Q\left(\left[\tilde{e}_{l_{k}}, \tilde{e}_{\iota_{l}}\right], \tilde{e}_{l_{m}}\right)^{2}
$$

for $m, k, l=1, \ldots, n$. The sum here is taken over all $\iota_{k}, \iota_{l}$, and $\iota_{m}$ such that $\tilde{e}_{l_{k}} \in \mathfrak{p}_{k}, \tilde{e}_{l_{l}} \in \mathfrak{p}_{l}$, and $\tilde{e}_{l_{m}} \in \mathfrak{p}_{m}$. Note that $\gamma_{k, l}^{m}$ is independent of the choice of $\left(\tilde{e}_{i}\right)_{i=1}^{d-1}$.

Consider a Riemannian metric $G$ on $M$. Suppose that $h, f_{1}, \ldots, f_{n}$ are smooth functions from $[0, \sigma]$ to $(0, \infty)$. Let $G$ be defined by the equality

$$
G=h^{2}(r) \mathrm{d} r \otimes \mathrm{d} r+G_{r}, \quad r \in[0, \sigma] .
$$

The tensor field $G_{r}$ in the right-hand side is the $\mathcal{G}$-invariant Riemannian metric on $\mathcal{G} / \mathcal{H}$ such that

$$
G_{r}(X, Y)=f_{1}^{2}(r) Q\left(\operatorname{pr}_{\mathfrak{p}_{1}} X, \operatorname{pr}_{\mathfrak{p}_{1}} Y\right)+\cdots+f_{n}^{2}(r) Q\left(\operatorname{pr}_{\mathfrak{p}_{n}} X, \operatorname{pr}_{\mathfrak{p}_{n}} Y\right), \quad X, Y \in \mathfrak{p} .
$$

In the sequel, the prime next to a real-valued function on $[0, \sigma]$ will denote the derivative of this function. 
Lemma 3.1 The Ricci curvature of the Riemannian metric G given by (3.1) and (3.2) obeys the equality

$$
\operatorname{Ric}(G)=\operatorname{Ric}^{\text {lin }}+\operatorname{Ric}_{r}^{\text {orb }}, \quad r \in[0, \sigma],
$$

where $\operatorname{Ric}^{\text {lin }}$ is the $(0,2)$-tensor field on $[0, \sigma]$ satisfying

$$
\operatorname{Ric}^{\operatorname{lin}}=-\sum_{k=1}^{n} d_{k}\left(\frac{f_{k}^{\prime \prime}}{f_{k}}-\frac{h^{\prime} f_{k}^{\prime}}{h f_{k}}\right) d r \otimes d r
$$

and $\mathrm{Ric}_{r}^{\text {orb }}$ is the $\mathcal{G}$-invariant $(0,2)$-tensor field on $\mathcal{G} / \mathcal{H}$ satisfying

$$
\begin{aligned}
\operatorname{Ric}_{r}^{\text {orb }}(X, Y)=\sum_{i=1}^{n}\left(\frac{\beta_{i}}{2}\right. & +\sum_{k, l=1}^{n} \gamma_{i, k}^{l} \frac{f_{i}^{4}-2 f_{k}^{4}}{4 f_{k}^{2} f_{l}^{2}} \\
& \left.\quad-\frac{f_{i} f_{i}^{\prime}}{h} \sum_{k=1}^{n} d_{k} \frac{f_{k}^{\prime}}{h f_{k}}+\frac{f_{i}^{\prime 2}}{h^{2}}-\frac{f_{i} f_{i}^{\prime \prime}}{h^{2}}+\frac{f_{i} h^{\prime} f_{i}^{\prime}}{h^{3}}\right) Q\left(\operatorname{pr}_{\mathfrak{p}_{i}} X, \operatorname{pr}_{\mathfrak{p}_{i}} Y\right)
\end{aligned}
$$

for $X, Y \in \mathfrak{p}$.

Proof The terms involving $\mathrm{d} r$ are computed and listed under Proposition 1.14 in [18]. Let us find $\mathrm{Ric}_{r}^{\text {orb }}$. Hypothesis 2.1 implies

$$
\operatorname{Ric}_{r}^{\text {orb }}(X, Y)=0
$$

when $X \in \mathfrak{p}_{i}$ and $Y \in \mathfrak{p}_{j}$ for $i, j=1, \ldots, n$ such that $i \neq j$. Remark 1.16 in [18] states that

$$
\begin{aligned}
\operatorname{Ric}_{r}^{\text {orb }}(X, X)=\left(\frac{\beta_{i}}{2}\right. & +\sum_{k, l=1}^{n} \gamma_{i, k}^{l} \frac{f_{i}^{4}-2 f_{k}^{4}}{4 f_{k}^{2} f_{l}^{2}} \\
& \left.\quad-\frac{f_{i} f_{i}^{\prime}}{h} \sum_{k=1}^{n} d_{k} \frac{f_{k}^{\prime}}{h f_{k}}+\frac{f_{i}^{\prime 2}}{h^{2}}-\frac{f_{i} f_{i}^{\prime \prime}}{h^{2}}+\frac{f_{i} h^{\prime} f_{i}^{\prime}}{h^{3}}\right) Q(X, X)
\end{aligned}
$$

when $X \in \mathfrak{p}_{i}$ and $i=1, \ldots, n$. In view of Hypothesis 2.1, the desired expression for Ric ${ }_{r}^{\text {orb }}$ immediately follows.

If the Ricci curvature of $G$ coincides with $T$, then Lemma 3.1 yields the equalities

$$
\begin{aligned}
& -\sum_{k=1}^{n} d_{k}\left(\frac{f_{k}^{\prime \prime}}{f_{k}}-\frac{h^{\prime} f_{k}^{\prime}}{h f_{k}}\right)=1, \\
& \frac{\beta_{i}}{2}+\sum_{k, l=1}^{n} \gamma_{i, k}^{l} \frac{f_{i}^{4}-2 f_{k}^{4}}{4 f_{k}^{2} f_{l}^{2}}-\frac{f_{i} f_{i}^{\prime}}{h} \sum_{k=1}^{n} d_{k} \frac{f_{k}^{\prime}}{h f_{k}}+\frac{f_{i}^{\prime 2}}{h^{2}}-\frac{f_{i} f_{i}^{\prime \prime}}{h^{2}}+\frac{f_{i} h^{\prime} f_{i}^{\prime}}{h^{3}}=\phi_{i}, \quad i=1, \ldots, n .
\end{aligned}
$$

The following result is essentially a restatement of the contracted second Bianchi identity.

Lemma 3.2 Assume that the Ricci curvature of the metric G given by (3.1) and (3.2) obeys the equality

$$
\operatorname{Ric}(G)=\bar{\sigma}(r) d r \otimes d r+T_{r}, \quad r \in[0, \sigma],
$$


with $\bar{\sigma}$ being a smooth function on $[0, \sigma]$ and $T_{r}$ satisfying (2.4). Then,

$$
\frac{\bar{\sigma}^{\prime}}{2 h^{2}}-\frac{\bar{\sigma} h^{\prime}}{h^{3}}=\sum_{k=1}^{n} d_{k}\left(\frac{\phi_{k}^{\prime}}{2 f_{k}^{2}}-\frac{\bar{\sigma} f_{k}^{\prime}}{h^{2} f_{k}}\right) \text {. }
$$

Proof Fix a $Q$-orthonormal basis $\left(\tilde{e}_{i}\right)_{i=1}^{d-1}$ of the space $\mathfrak{p}$ adapted to the decomposition (2.1). Recall that we identify $\mathfrak{p}$ with the tangent space of $\mathcal{G} / \mathcal{H}$ at $\mathcal{H}$. Given $r_{0} \in[0, \sigma]$, let us construct a $\mathcal{G}$-invariant $G$-orthonormal frame field $\left(e_{i}\right)_{i=1}^{d}$ on a neighbourhood $U$ of $\left(r_{0}, \mathcal{H}\right)$ in $M$ so that the following requirements are met:

1. The equality $e_{i}=\left(0, \frac{1}{f_{i}(r)} \tilde{e}_{i}\right)$ holds at $(r, \mathcal{H})$ for every $i=1, \ldots, d-1$ as long as $(r, \mathcal{H}) \in U$.

2. The vector field $e_{d}$ coincides with $\left(\frac{1}{h(r)} \frac{\partial}{\partial r}, 0\right)$ on $U$.

The contracted second Bianchi identity then implies

$$
\sum_{i=1}^{d} \nabla_{e_{i}} \operatorname{Ric}(G)\left(e_{i}, e_{d}\right)=\frac{1}{2} e_{d}\left(\sum_{i=1}^{d} \operatorname{Ric}(G)\left(e_{i}, e_{i}\right)\right) .
$$

The symbol $\nabla$ in the left-hand side denotes the covariant derivative in the tensor bundle over $M$ given by the Levi-Civita connection of $G$. We calculate and see that the equalities

$$
\begin{aligned}
\sum_{i=1}^{d} \nabla_{e_{i}} \operatorname{Ric}(G)\left(e_{i}, e_{d}\right)= & \sum_{i=1}^{d} e_{i}\left(\operatorname{Ric}(G)\left(e_{i}, e_{d}\right)\right)-\sum_{i=1}^{d} \operatorname{Ric}(G)\left(\nabla_{e_{i}} e_{i}, e_{d}\right) \\
& -\sum_{i=1}^{d} \operatorname{Ric}(G)\left(e_{i}, \nabla_{e_{i}} e_{d}\right) \\
= & e_{d}\left(\operatorname{Ric}(G)\left(e_{d}, e_{d}\right)\right)-\sum_{i=1}^{d} G\left(\nabla_{e_{i}} e_{i}, e_{d}\right) \operatorname{Ric}(G)\left(e_{d}, e_{d}\right) \\
& -\sum_{i=1}^{d} G\left(\nabla_{e_{i}} e_{d}, e_{i}\right) \operatorname{Ric}(G)\left(e_{i}, e_{i}\right) \\
= & \frac{\bar{\sigma}^{\prime}}{h^{3}}-\frac{2 \bar{\sigma} h^{\prime}}{h^{4}}+\sum_{k=1}^{n} d_{k} \frac{\bar{\sigma} f_{k}^{\prime}}{h^{3} f_{k}}-\sum_{k=1}^{n} d_{k} \frac{f_{k}^{\prime}}{h f_{k}^{3}} \phi_{k},
\end{aligned}
$$

as well as the equality

$$
\frac{1}{2} e_{d}\left(\sum_{i=1}^{d} \operatorname{Ric}(G)\left(e_{i}, e_{i}\right)\right)=\sum_{k=1}^{n} d_{k}\left(\frac{\phi_{k}^{\prime}}{2 h f_{k}^{2}}-\frac{f_{k}^{\prime}}{h f_{k}^{3}} \phi_{k}\right)+\frac{\bar{\sigma}^{\prime}}{2 h^{3}}-\frac{\bar{\sigma} h^{\prime}}{h^{4}}
$$

hold at $\left(r_{0}, \mathcal{H}\right)$. The assertion of the lemma follows immediately.

Denote by $f$ and $\phi$ the functions $\left(f_{1}, \ldots, f_{n}\right)$ and $\left(\phi_{1}, \ldots, \phi_{n}\right)$ acting from $[0, \sigma]$ to $(0, \infty)^{n}$. We can rewrite the second equality in (3.3) as

$$
f^{\prime \prime}(r)=F\left(h(r), h^{\prime}(r), f(r), f^{\prime}(r), \phi(r)\right), \quad r \in[0, \sigma],
$$


with $F:(0, \infty) \times \mathbb{R} \times(0, \infty)^{n} \times \mathbb{R}^{n+n} \rightarrow \mathbb{R}^{n}$ given by the formulas

$$
\begin{aligned}
& F(p, q, x, y, z)=\left(F_{1}(p, q, x, y, z), \ldots, F_{n}(p, q, x, y, z)\right), \\
& F_{i}(p, q, x, y, z)=\frac{\beta_{i} p^{2}}{2 x_{i}}+p^{2} \sum_{k, l=1}^{n} \gamma_{i, k}^{l} \frac{x_{i}^{4}-2 x_{k}^{4}}{4 x_{i} x_{k}^{2} x_{l}^{2}} \\
& -\sum_{k=1}^{n} d_{k} \frac{y_{i} y_{k}}{x_{k}}+\frac{y_{i}^{2}}{x_{i}}+\frac{q y_{i}}{p}-\frac{p^{2}}{x_{i}} z_{i}, \quad i=1, \ldots, n, \\
& p \in(0, \infty), q \in \mathbb{R}, \quad x=\left(x_{1}, \ldots, x_{n}\right) \in(0, \infty)^{n}, \\
& y=\left(y_{1}, \ldots, y_{n}\right) \in \mathbb{R}^{n}, \quad z=\left(z_{1}, \ldots, z_{n}\right) \in \mathbb{R}^{n} .
\end{aligned}
$$

The prime next to a vector-valued function means component-wise differentiation. Combining the two equalities in (3.3), we find

$$
H_{1}\left(f(r), f^{\prime}(r)\right)=h^{2}(r) H_{2}(f(r), \phi(r)), \quad r \in[0, \sigma],
$$

with the mappings $H_{1}:(0, \infty)^{n} \times \mathbb{R}^{n} \rightarrow \mathbb{R}$ and $H_{2}:(0, \infty)^{n} \times \mathbb{R}^{n} \rightarrow \mathbb{R}$ defined by the formulas

$$
\begin{aligned}
H_{1}(x, y) & =1-\sum_{k=1}^{n} d_{k}\left(\sum_{l=1}^{n} d_{l} \frac{y_{k} y_{l}}{x_{k} x_{l}}-\frac{y_{k}^{2}}{x_{k}^{2}}\right), \\
H_{2}(x, z) & =\sum_{k=1}^{n} d_{k}\left(\frac{z_{k}}{x_{k}^{2}}-\frac{\beta_{k}}{2 x_{k}^{2}}-\sum_{l, m=1}^{n} \gamma_{k, l}^{m} \frac{x_{k}^{4}-2 x_{l}^{4}}{4 x_{k}^{2} x_{l}^{2} x_{m}^{2}}\right), \\
x & =\left(x_{1}, \ldots, x_{n}\right) \in(0, \infty)^{n}, y=\left(y_{1}, \ldots, y_{n}\right) \in \mathbb{R}^{n}, z=\left(z_{1}, \ldots, z_{n}\right) \in \mathbb{R}^{n} .
\end{aligned}
$$

It will be convenient for us to denote

$$
H(x, y, z)=\sqrt{H_{1}(x, y) H_{2}^{-1}(x, z)}
$$

for $x \in(0, \infty)^{n}, y \in \mathbb{R}^{n}$, and $z \in \mathbb{R}^{n}$ such that $H_{2}(x, z) \neq 0$ and $H_{1}(x, y) H_{2}^{-1}(x, z) \geq 0$.

Solving (3.4) for $h^{\prime}(r)$ and substituting 1 for $\bar{\sigma}(r)$, we arrive at the following conclusion: if $\operatorname{Ric}(G)$ coincides with $T$, then

$$
h^{\prime}(r)=K\left(h(r), f(r), f^{\prime}(r), \phi^{\prime}(r)\right), \quad r \in[0, \sigma] .
$$

Here, $K:(0, \infty)^{1+n} \times \mathbb{R}^{n+n} \rightarrow \mathbb{R}$ is given by

$$
\begin{aligned}
& K(p, x, y, w)=\sum_{i=1}^{n} d_{i}\left(\frac{p y_{i}}{x_{i}}-\frac{p^{3} w_{i}}{2 x_{i}^{2}}\right), \\
& p \in(0, \infty), \quad x=\left(x_{1}, \ldots, x_{n}\right) \in(0, \infty)^{n}, \\
& y=\left(y_{1}, \ldots, y_{n}\right) \in \mathbb{R}^{n}, \quad w=\left(w_{1}, \ldots, w_{n}\right) \in \mathbb{R}^{n} .
\end{aligned}
$$

Let $a$ and $b$ denote the vectors $\left(a_{1}, \ldots, a_{n}\right)$ and $\left(b_{1}, \ldots, b_{n}\right)$ with the numbers $a_{1}, \ldots, a_{n}$ and $b_{1}, \ldots, b_{n}$ coming from (2.6). If the metric $G_{\partial M}$ induced by $G$ on $\partial M$ equals $R$, then

$$
f(0)=a, \quad f(\sigma)=b .
$$

We also point out that whenever (3.6) holds, we must have

$$
H_{1}\left(f(0), f^{\prime}(0)\right)=h^{2}(0) H_{2}(f(0), \phi(0)) .
$$




\subsection{Proof of Theorem 2.2 (less the key lemma)}

Intuitively, our plan for proving Theorem 2.2 is to find a metric $G$ satisfying two requirements. The first one is that $\operatorname{Ric}(G)$ equal $T$ in the directions tangent to the $\mathcal{G}$-orbits. The other is that $G$ and $T$ obey the contracted second Bianchi identity. When both of these requirements are met, it must be the case that $\operatorname{Ric}(G)=T$. We define $\rho_{0}$ by the formula

$$
\rho_{0}(p, q)=2 \sum_{k=1}^{n} d_{k}\left(\frac{\beta_{k} q^{2}}{2 p^{2}}+\sum_{l, m=1}^{n} \gamma_{k, l}^{m} \frac{q^{6}}{4 p^{6}}\right), \quad p, q \in(0, \infty)
$$

Lemma 3.3 Assume that inequalities (2.9) and the first inequality in (2.10) are satisfied. There exists a function $\sigma_{0}:(0, \infty)^{3} \rightarrow(0, \infty)$ such that the following statement holds: if $\sigma$ is less than $\sigma_{0}\left(\alpha, \omega_{1}, \omega_{2}\right)$, then we can find smooth $f:[0, \sigma] \rightarrow(0, \infty)^{n}$ and $h:[0, \sigma] \rightarrow$ $(0, \infty)$ solving Eqs. (3.5) and (3.7) under the boundary conditions (3.8) and (3.9).

We will present the proof of Lemma 3.3 in Sect. 3.3. Meanwhile, fix a function $\sigma_{0}$ satisfying the assertion of this lemma. Suppose that $\sigma$ is less than $\sigma_{0}\left(\alpha, \omega_{1}, \omega_{2}\right)$. Let $f:[0, \sigma] \rightarrow$ $(0, \infty)^{n}$ and $h:[0, \sigma] \rightarrow(0, \infty)$ be smooth functions obeying (3.5), (3.7), (3.8) and (3.9). We define the metric $G$ on $M$ through (3.1)-(3.2). It is easy to see that the Ricci curvature of $G$ must equal

$$
\bar{\sigma}(r) \mathrm{d} r \otimes \mathrm{d} r+T_{r}, \quad r \in[0, \sigma],
$$

for some $\bar{\sigma}:[0, \sigma] \rightarrow \mathbb{R}$. The induced metric $G_{\partial M}$ coincides with $R$. The proof of Theorem 2.2 will be complete if we demonstrate that $\bar{\sigma}(r)=1$ for all $r \in[0, \sigma]$.

Lemma 3.2 implies

$$
\bar{\sigma}^{\prime}=\frac{2 \bar{\sigma} h^{\prime}}{h}+\sum_{i=1}^{n} d_{i}\left(\frac{h^{2} \phi_{i}^{\prime}}{f_{i}^{2}}-\frac{2 \bar{\sigma} f_{i}^{\prime}}{f_{i}}\right) .
$$

Thanks to (3.7), this formula will still hold if we replace $\bar{\sigma}$ in it by the function identically equal to 1 on $[0, \sigma]$. Furthermore, invoking Lemma 3.1 and the boundary conditions (3.8)(3.9), we find

$$
\begin{aligned}
\bar{\sigma}(0)=-\sum_{k=1}^{n} d_{k}\left(\frac{f_{k}^{\prime \prime}(0)}{a_{k}}-\frac{h^{\prime}(0) f_{k}^{\prime}(0)}{h(0) a_{k}}\right) \\
=-\sum_{k=1}^{n} d_{k}\left(\frac{\beta_{k} h^{2}(0)}{2 a_{k}^{2}}+h^{2}(0) \sum_{l, m=1}^{n} \gamma_{k, l}^{m} \frac{a_{k}^{4}-2 a_{l}^{4}}{4 a_{k}^{2} a_{l}^{2} a_{m}^{2}}\right. \\
\left.-\sum_{l=1}^{n} d_{l} \frac{f_{k}^{\prime}(0) f_{l}^{\prime}(0)}{a_{k} a_{l}}+\frac{f_{k}^{\prime 2}(0)}{a_{k}^{2}}-\frac{h^{2}(0)}{a_{k}^{2}} \phi_{k}(0)\right) \\
=h^{2}(0) H_{2}(a, \phi(0))+\left(1-H_{1}\left(a, f^{\prime}(0)\right)\right)=1 .
\end{aligned}
$$

The standard theorems on the uniqueness of solutions to ordinary differential equations now yield $\bar{\sigma}(r)=1$ for $r \in[0, \sigma]$. 


\subsection{Proof of Lemma 3.3}

From now on and until the end of Sect. 3.3, we assume that inequalities (2.9) and the first inequality in (2.10) are satisfied. Let $\bar{f}:[0, \sigma] \rightarrow \mathbb{R}^{n}$ be defined by

$$
\bar{f}(r)=a \frac{\sigma-r}{\sigma}+b \frac{r}{\sigma}, \quad r \in[0, \sigma] .
$$

We seek the function $f$, whose existence Lemma 3.3 asserts, in a neighbourhood of $\bar{f}$. This, in particular, will help us ensure the positivity of the components of $f$. Similarly, we look for the function $h$ in a neighbourhood of the function $\bar{h}$ to be introduced in Lemma 3.4. Our arguments will involve the constants

$$
\begin{aligned}
& H_{0}=H\left(\bar{f}(0), \bar{f}^{\prime}(0), \phi(0)\right), \\
& K_{0}=\sup _{p \in\left[\frac{H_{0}}{2}, \frac{3 H_{0}}{2}\right]} \sup _{r \in[0, \sigma]}\left|K\left(p, \bar{f}(r), \bar{f}^{\prime}(r), \phi^{\prime}(r)\right)\right| .
\end{aligned}
$$

The second inequality in (2.9) and the first inequality in (2.10) imply that $H_{0}$ is well defined under the assumptions of Lemma 3.4. Recall that the letter $d$ stands for the dimension of $M$. It is evident that $\sum_{i=1}^{n} d_{i}=d-1$.

Lemma 3.4 Let $\rho_{1}, \sigma_{1}>0$ be given by the formulas

$$
\begin{aligned}
& \rho_{1}=\max \left\{4\left(\sum_{k=1}^{n} d_{k}\left(\frac{\alpha}{\omega_{1}^{2}}+\sum_{l, m=1}^{n} \gamma_{k, l}^{m} \frac{\omega_{2}^{4}}{2 \omega_{1}^{6}}\right)\right)^{\frac{1}{2}}, \frac{9}{4}\left(\frac{\rho_{0}\left(\omega_{1}, \omega_{2}\right)}{2 \omega_{2}^{2}}\right)^{-\frac{1}{2}}\right\}, \\
& \sigma_{1}=\min \left\{1, \frac{\omega_{1}}{4 d}, \frac{2 \omega_{1}^{2}}{\left(2 \rho_{1}^{2} \omega_{1}+\rho_{1}^{4}\right)(d-1)}\right\} .
\end{aligned}
$$

If $\sigma \leq \sigma_{1}$, then the problem

$$
\begin{aligned}
\bar{h}^{\prime}(r) & =K\left(\bar{h}(r), \bar{f}(r), \bar{f}^{\prime}(r), \phi^{\prime}(r)\right), \quad r \in[0, \sigma], \\
\bar{h}(0) & =H_{0},
\end{aligned}
$$

has a unique smooth solution $\bar{h}:[0, \sigma] \rightarrow\left(\frac{1}{\rho_{1}}, \rho_{1}\right)$.

Proof Assume that $\sigma<\sigma_{1}$. Employing the standard theory of ordinary differential equations, it is easy to show that the problem (3.11) has a unique smooth solution on the interval $\left[0, \min \left\{\sigma, \frac{H_{0}}{2 \varkappa}\right\}\right]$ as long as $K_{0} \leq \varkappa$. The values of this solution must lie in $\left[\frac{H_{0}}{2}, \frac{3 H_{0}}{2}\right]$.

Our assumptions imply

$$
\frac{1}{\rho_{1}} \leq \frac{H_{0}}{2}<\frac{3 H_{0}}{2} \leq \rho_{1}
$$

In view of (2.9), the estimate

$$
K_{0} \leq \frac{\left(2 \rho_{1} \omega_{1} \sigma+\rho_{1}^{3} \sigma\right)(d-1)}{2 \omega_{1}^{2}}
$$

holds true. Keeping these facts in mind, we conclude that problem (3.11) has a unique smooth solution

$$
\bar{h}:\left[0, \min \left\{\sigma, \frac{2 \omega_{1}^{2}}{\left(2 \rho_{1}^{2} \omega_{1} \sigma+\rho_{1}^{4} \sigma\right)(d-1)}\right\}\right] \rightarrow\left(\frac{1}{\rho_{1}}, \rho_{1}\right) .
$$


At the same time, whenever $\sigma \leq \sigma_{1}$, the equality

$$
\sigma=\min \left\{\sigma, \frac{2 \omega_{1}^{2}}{\left(2 \rho_{1}^{2} \omega_{1} \sigma+\rho_{1}^{4} \sigma\right)(d-1)}\right\}
$$

is satisfied. This means $\bar{h}$ is actually defined on $[0, \sigma]$.

From this moment on and until the end of Sect. 3.3, let us assume that $\sigma \leq \sigma_{1}$. It then makes sense to talk about $\bar{h}$. Our plan is to prove, for small $\sigma$, the existence of smooth $u:[0, \sigma] \rightarrow \mathbb{R}^{n}$ and $v:[0, \sigma] \rightarrow \mathbb{R}$ solving the equations

$$
\begin{aligned}
& u^{\prime \prime}(r)=F\left(\bar{h}(r)+v(r), \bar{h}^{\prime}(r)+v^{\prime}(r), \bar{f}(r)+u(r), \bar{f}^{\prime}(r)+u^{\prime}(r), \phi(r)\right), \\
& v^{\prime}(r)=-\bar{h}^{\prime}(r)+K\left(\bar{h}(r)+v(r), \bar{f}(r)+u(r), \bar{f}^{\prime}(r)+u^{\prime}(r), \phi^{\prime}(r)\right), \quad r \in[0, \sigma],
\end{aligned}
$$

under the boundary conditions

$$
\begin{aligned}
& u(0)=u(\sigma)=0, \\
& v(0)=-\bar{h}(0)+H\left(\bar{f}(0)+u(0), \bar{f}^{\prime}(0)+u^{\prime}(0), \phi(0)\right) .
\end{aligned}
$$

We will then set $f=\bar{f}+u$ and $h=\bar{h}+v$. It is obvious that these functions will obey (3.5), (3.7), (3.8) and (3.9).

Our proof of the existence of $u$ and $v$ will rely on the Schauder fixed point theorem. Let us introduce the space $\mathcal{B}$ of all the pairs $\left(v_{1}, v_{2}\right)$ such that $v_{1}:[0, \sigma] \rightarrow \mathbb{R}^{n}$ is $C^{1}$-differentiable and $v_{2}:[0, \sigma] \rightarrow \mathbb{R}$ is continuous. We endow $\mathcal{B}$ with the norm

$$
\left|\left(v_{1}, v_{2}\right)\right|_{\mathcal{B}}=\sup _{r \in[0, \sigma]}\left|v_{1}(r)\right|_{\mathbb{R}^{n}}+\sigma \sup _{r \in[0, \sigma]}\left|v_{1}^{\prime}(r)\right|_{\mathbb{R}^{n}}+\sup _{r \in[0, \sigma]}\left|v_{2}(r)\right|,
$$

where $|\cdot|_{\mathbb{R}^{n}}$ is the Euclidean norm in $\mathbb{R}^{n}$. Denote by $B(L)$ the closed ball in $\mathcal{B}$ of radius $L>0$ centred at 0 . We will now define a map $\mathcal{C}: B(L) \rightarrow \mathcal{B}$ and show that $\mathcal{C}$ has a fixed point $(u, v)$ under appropriate conditions. The functions $u$ and $v$ will satisfy (3.12) and (3.13).

Assume that the radius $L$ is less than or equal to $\frac{\sigma}{2} \min \left\{\omega_{1}, \frac{1}{\rho_{1}}\right\}$. Given $(\mu, \nu) \in B(L)$, let $u_{\mu, v}$ be the unique solution of the problem

$$
\begin{aligned}
& u_{\mu, v}^{\prime \prime}(r)=\bar{F}\left(\bar{h}(r)+v(r), \bar{f}(r)+\mu(r), \bar{f}^{\prime}(r)+\mu^{\prime}(r), \phi(r), \phi^{\prime}(r)\right), \quad r \in[0, \sigma], \\
& u_{\mu, v}(0)=u_{\mu, v}(\sigma)=0,
\end{aligned}
$$

where

$\bar{F}(p, x, y, z, w)=F(p, K(p, x, y, w), x, y, z), \quad p \in(0, \infty), x \in(0, \infty)^{n}, y, z, w \in \mathbb{R}^{n}$.

It is obvious that such a solution exists. Moreover, it is easy to write down an explicit formula for it (the formula is quite lengthy, and we will not present it here; the reader may find it in, e.g. [20, Section XII.4]). We will set $\mathcal{C}(\mu, v)=\left(u_{\mu, \nu}, v_{\mu, v}\right)$ for a properly chosen $v_{\mu, \nu}:[0, \sigma] \rightarrow \mathbb{R}$. Before we can describe $v_{\mu, \nu}$, however, we need to state the following auxiliary result.

Lemma 3.5 Let $\Theta$ be given by the formula

$$
\Theta=\sqrt{d} \max _{i=1, \ldots, n}\left|\frac{4 \beta_{i} \rho_{1}^{2}}{\omega_{1}}+1536 \rho_{1}^{2} \sum_{k, l=1}^{n} \gamma_{i, k}^{l} \frac{\omega_{2}^{4}}{\omega_{1}^{5}}+2 \omega_{1}+\left(2 \omega_{1}+2 \omega_{1}^{2}+8 \rho_{1}^{2}\right)(d-1)+\frac{8 \alpha \rho_{1}^{2}}{\omega_{1}}\right| .
$$


If $(\mu, v)$ lie in $B(L)$, then the estimate

$$
\sup _{r \in[0, \sigma]}\left|\bar{F}\left(\bar{h}(r)+v(r), \bar{f}(r)+\mu(r), \bar{f}^{\prime}(r)+\mu^{\prime}(r), \phi(r), \phi^{\prime}(r)\right)\right|_{\mathbb{R}^{n}} \leq \Theta
$$

holds true. Moreover, in this case, we have

$$
\left|u_{\mu, v}(r)\right|_{\mathbb{R}^{n}} \leq \frac{\sigma^{2}}{8} \Theta, \quad\left|u_{\mu, v}^{\prime}(r)\right|_{\mathbb{R}^{n}} \leq \frac{\sigma}{2} \Theta, \quad r \in[0, \sigma] .
$$

Proof Inequality (3.15) is a straightforward consequence of the definition of $\bar{F}$. To obtain (3.16), it suffices to write down an explicit formula for $u_{\mu, v}$ and perform elementary estimation of its terms [for the second part of (3.16), one needs to differentiate before estimating]. We refer to [20, Section XII.4] for the details of this argument.

From now on and until the end of Sect. 3.3, we assume

$$
\sigma \leq \min \left\{\sigma_{1}, \sqrt{\frac{\omega_{1}}{\Theta}}, \frac{\omega_{1}}{2 d \Theta}\right\} .
$$

Given $(\mu, v) \in B(L)$, let us introduce $v_{\mu, v}:[0, \sigma] \rightarrow \mathbb{R}$ through the formula

$$
\begin{aligned}
v_{\mu, v}(r)= & -\bar{h}(0)+H\left(\bar{f}(0)+u_{\mu, v}(0), \bar{f}^{\prime}(0)+u_{\mu, v}^{\prime}(0), \phi(0)\right) \\
& +\int_{0}^{r}\left(-\bar{h}^{\prime}(s)+K\left(\bar{h}(s)+v(s), \bar{f}(s)+u_{\mu, v}(s), \bar{f}^{\prime}(s)\right.\right. \\
& \left.\left.+u_{\mu, v}^{\prime}(s), \phi^{\prime}(s)\right)\right) \mathrm{d} s, \quad r \in[0, \sigma] .
\end{aligned}
$$

Lemma 3.5 and inequality (3.17) imply the estimates

$$
\sup _{r \in[0, \sigma]}\left|u_{\mu, v}(r)\right|_{\mathbb{R}_{n}} \leq \frac{\omega_{1}}{2}, \sup _{r \in[0, \sigma]}\left|u_{\mu, v}^{\prime}(r)\right|_{\mathbb{R}_{n}} \leq \frac{\omega_{1}}{4 d},
$$

which ensure that the right-hand side of (3.18) is well defined (indeed, the expression $H(\bar{f}(0), y, \phi(0))$ is well defined and positive whenever $\left.|y|_{\mathbb{R}^{n}} \leq \frac{\omega_{1}}{2 d}\right)$. We now $\operatorname{set} \mathcal{C}(\mu, v)=$ $\left(u_{\mu, \nu}, v_{\mu, \nu}\right)$. Our next goal is to show that when $\sigma$ is sufficiently small and the radius $L$ is appropriately chosen, the map $\mathcal{C}$ satisfies the conditions of the Schauder theorem.

Suppose that $\theta>0$ is a constant obeying the inequalities

$$
\begin{aligned}
|H(x, y, z)-H(x, \hat{y}, z)| & \leq \theta|y-\hat{y}|_{\mathbb{R}^{n}} \\
|H(x, y, z)-H(x, \hat{y}, z)| & \leq \theta \sum_{k, l=1}^{n}\left|y_{k} y_{l}-\hat{y}_{k} \hat{y}_{l}\right|, \\
x & \in\left[\omega_{1}, \omega_{2}\right]^{n}, \\
y & =\left(y_{1}, \ldots, y_{n}\right) \in\left[-\frac{\omega_{1}}{2 d}, \frac{\omega_{1}}{2 d}\right]^{n}, \\
\hat{y} & =\left(\hat{y}_{1}, \ldots, \hat{y}_{n}\right) \in\left[-\frac{\omega_{1}}{2 d}, \frac{\omega_{1}}{2 d}\right]^{n}, \\
z & \in\left\{\left(z_{1}, \ldots, z_{n}\right) \in[0, \alpha]^{n} \mid \sum_{k=1}^{n} d_{k} z_{k} \geq \rho_{0}\left(\omega_{1}, \omega_{2}\right)\right\},
\end{aligned}
$$


and the inequality

$$
\begin{aligned}
& |K(p, x, y, w)-K(\hat{p}, \hat{x}, \hat{y}, w)| \leq \theta\left(|p-\hat{p}|+|x-\hat{x}|_{\left.\mathbb{R}^{n}+|y-\hat{y}|_{\mathbb{R}^{n}}\right),}\right. \\
& \quad p, \hat{p} \in\left[\frac{1}{2 \rho_{1}}, 2 \rho_{1}\right], x, \hat{x} \in\left[\frac{\omega_{1}}{2}, 2 \omega_{2}\right]^{n}, y, \hat{y} \in\left[-\frac{\omega_{1}}{2 d}, \frac{\omega_{1}}{2 d}\right]^{n}, w \in[-1,1]^{n} .
\end{aligned}
$$

It is obvious that such a $\theta$ exists. We define

$$
\begin{gathered}
\Sigma=\Theta+\theta n^{2}\left(2 \Theta+\Theta^{2}+\omega_{1}\right), \\
\sigma_{0}\left(\alpha, \omega_{1}, \omega_{2}\right)=\min \left\{\sigma_{1}, \sqrt{\frac{\omega_{1}}{\Theta}}, \frac{\omega_{1}}{2 d \Theta}, \frac{\omega_{1}}{2 \Sigma}, \frac{1}{2 \rho_{1} \Sigma}\right\} .
\end{gathered}
$$

Note that $\sigma_{0}$ is the function whose existence (along with $\rho_{0}$ ) Lemma 3.3 asserts. Let us also set $L_{0}=\sigma^{2} \Sigma$. From now on, we will assume that the second inequality in (2.10) holds, i.e. $\sigma<\sigma_{0}\left(\alpha, \omega_{1}, \omega_{2}\right)$. This implies, in particular, that $L_{0}$ cannot exceed $\frac{\sigma}{2} \min \left\{\omega_{1}, \frac{1}{\rho_{1}}\right\}$.

Lemma 3.6 The image $\mathcal{C} B\left(L_{0}\right)$ is contained in $B\left(L_{0}\right)$.

Proof Take a pair $(\mu, v)$ from $B\left(L_{0}\right)$. Our goal is to show that $\mathcal{C}(\mu, v)$ lies in $B\left(L_{0}\right)$. Lemma 3.5 yields the estimate

$$
\left|\left(u_{\mu, v}, v_{\mu, v}\right)\right|_{\mathcal{B}} \leq \sigma^{2} \Theta+\sup _{r \in[0, \sigma]}\left|v_{\mu, v}(r)\right| .
$$

Remembering the second formula in (2.9), we also find

$$
\begin{aligned}
\left|v_{\mu, v}(r)\right| \leq & \left|-\bar{h}(0)+H\left(\bar{f}(0)+u_{\mu, v}(0), \bar{f}^{\prime}(0)+u_{\mu, v}^{\prime}(0), \phi(0)\right)\right| \\
& +\sigma \sup _{s \in[0, r]}\left|-\bar{h}^{\prime}(s)+K\left(\bar{h}(s)+v(s), \bar{f}(s)+u_{\mu, v}(s), \bar{f}^{\prime}(s)+u_{\mu, v}^{\prime}(s), \phi^{\prime}(s)\right)\right| \\
= & \left|H\left(\bar{f}(0), \bar{f}^{\prime}(0)+u_{\mu, v}^{\prime}(0), \phi(0)\right)-H\left(\bar{f}(0), \bar{f}^{\prime}(0), \phi(0)\right)\right| \\
& +\sigma \sup _{s \in[0, r]} \mid K\left(\bar{h}(s)+v(s), \bar{f}(s)+u_{\mu, v}(s), \bar{f}^{\prime}(s)+u_{\mu, v}^{\prime}(s), \phi^{\prime}(s)\right) \\
& -K\left(\bar{h}(s), \bar{f}(s), \bar{f}^{\prime}(s), \phi^{\prime}(s)\right) \mid \\
\leq & \theta \sum_{k, l=1}^{n}\left(\sigma\left|\left(u_{\mu, v}\right)_{k}^{\prime}(0)\right|+\sigma\left|\left(u_{\mu, v}\right)_{l}^{\prime}(0)\right|+\left|\left(u_{\mu, v}\right)_{k}^{\prime}(0)\left(u_{\mu, v}\right)_{l}^{\prime}(0)\right|\right) \\
& +\sigma \theta \sup _{s \in[0, r]}\left(|v(s)|+\left|u_{\mu, v}(s)\right|_{\mathbb{R}^{n}}+\left|u_{\mu, v}^{\prime}(s)\right|_{\mathbb{R}^{n}}\right) \\
\leq & \sigma^{2} \theta n^{2}\left(2 \Theta+\Theta^{2}+\omega_{1}\right), \quad r \in[0, \sigma],
\end{aligned}
$$

where $\left(u_{\mu, v}\right)_{k}$ and $\left(u_{\mu, v}\right)_{l}$ are the $k$ th and the $l$ th components of $u_{\mu, v}$. Consequently, it must be the case that

$$
\left|\left(u_{\mu, v}, v_{\mu, v}\right)\right|_{\mathcal{B}} \leq \sigma^{2}\left(\Theta+\theta n^{2}\left(2 \Theta+\Theta^{2}+\omega_{1}\right)\right)=\sigma^{2} \Sigma=L_{0} .
$$

Lemma 3.7 The map $\mathcal{C}: B\left(L_{0}\right) \rightarrow \mathcal{B}$ is continuous. 
Proof Without loss of generality, assume that the constant $\theta$ fixed above satisfies

$$
\begin{aligned}
& |\bar{F}(p, x, y, z, w)-\bar{F}(\hat{p}, \hat{x}, \hat{y}, z, w)|_{\mathbb{R}^{n}} \leq \theta\left(|p-\hat{p}|+|x-\hat{x}|_{\mathbb{R}^{n}}+|y-\hat{y}|_{\mathbb{R}^{n}}\right), \\
& \quad p, \hat{p} \in\left[\frac{1}{2 \rho_{1}}, 2 \rho_{1}\right], x, \hat{x} \in\left[\frac{\omega_{1}}{2}, 2 \omega_{2}\right]^{n}, y, \hat{y} \in\left[-\frac{\omega_{1}}{2}, \frac{\omega_{1}}{2}\right]^{n}, \\
& \quad z \in[0, \alpha]^{n}, w \in[-1,1]^{n} .
\end{aligned}
$$

Suppose that the pairs $\left(\mu_{1}, v_{1}\right)$ and $\left(\mu_{2}, v_{2}\right)$ lie in $B\left(L_{0}\right)$. The first formula in (3.14), along with inequalities (3.16) and (3.21), imply

$$
\begin{aligned}
& \sup _{r \in[0, \sigma]}\left|u_{\mu_{1}, v_{1}}(r)-u_{\mu_{2}, \nu_{2}}(r)\right|_{\mathbb{R}^{n}} \leq \frac{\sigma \theta}{8}\left|\left(\mu_{1}, v_{1}\right)-\left(\mu_{2}, v_{2}\right)\right|_{\mathcal{B}}, \\
& \sup _{r \in[0, \sigma]}\left|u_{\mu_{1}, v_{1}}^{\prime}(r)-u_{\mu_{2}, \nu_{2}}^{\prime}(r)\right|_{\mathbb{R}^{n}} \leq \frac{\theta}{2}\left|\left(\mu_{1}, v_{1}\right)-\left(\mu_{2}, v_{2}\right)\right|_{\mathcal{B}} .
\end{aligned}
$$

Using (3.18)-(3.20), we also find

$$
\begin{aligned}
\sup _{r \in[0, \sigma]}\left|v_{\mu_{1}, v_{1}}(r)-v_{\mu_{2}, \nu_{2}}(r)\right| \leq & \theta\left|u_{\mu_{1}, v_{1}}^{\prime}(0)-u_{\mu_{2}, \nu_{2}}^{\prime}(0)\right|_{\mathbb{R}^{n}}+\theta \int_{0}^{\sigma}\left|v_{1}(s)-v_{2}(s)\right| \mathrm{d} s \\
& +\theta \int_{0}^{\sigma}\left(\left|u_{\mu_{1}, v_{1}}(s)-u_{\mu_{2}, v_{2}}(s)\right|_{\mathbb{R}^{n}}\right. \\
& \left.+\left|u_{\mu_{1}, \nu_{1}}^{\prime}(s)-u_{\mu_{2}, \nu_{2}}^{\prime}(s)\right|_{\mathbb{R}^{n}}\right) \mathrm{d} s \\
\leq & \left(\frac{3 \theta^{2}}{2}+\theta\right)\left|\left(\mu_{1}, v_{1}\right)-\left(\mu_{2}, v_{2}\right)\right|_{\mathcal{B}} .
\end{aligned}
$$

Consequently, it must be the case that

$$
\left|\mathcal{C}\left(\mu_{1}, v_{1}\right)-\mathcal{C}\left(\mu_{2}, v_{2}\right)\right|_{\mathcal{B}} \leq\left(\frac{3 \theta^{2}}{2}+2 \theta\right)\left|\left(\mu_{1}, v_{1}\right)-\left(\mu_{2}, v_{2}\right)\right|_{\mathcal{B}},
$$

which tells us $\mathcal{C}$ is continuous.

Lemma 3.8 The closure of the $\operatorname{set} \mathcal{C} B\left(L_{0}\right)$ in $\mathcal{B}$ is a compact subset of $\mathcal{B}$.

Proof Suppose that $\left(\left(\mu_{j}, v_{j}\right)\right)_{j=1}^{\infty}$ are pairs from $B\left(L_{0}\right)$. It suffices to prove that the sequence $\left(\left(u_{\mu_{j}, v_{j}}, v_{\mu_{j}, v_{j}}\right)\right)_{j=1}^{\infty}$ has a convergent subsequence. The mean value theorem and Lemma 3.5 yield the estimates

$$
\begin{aligned}
&\left|u_{\mu_{j}, v_{j}}\left(r_{1}\right)-u_{\mu_{j}, v_{j}}\left(r_{2}\right)\right|_{\mathbb{R}^{n}} \leq \sup _{r \in[0, \sigma]}\left|u_{\mu_{j}, v_{j}}^{\prime}(r)\right|_{\mathbb{R}^{n}}\left|r_{1}-r_{2}\right| \leq \frac{\sigma}{2} \Theta\left|r_{1}-r_{2}\right|, \\
&\left|u_{\mu_{j}, v_{j}}^{\prime}\left(r_{1}\right)-u_{\mu_{j}, v_{j}}^{\prime}\left(r_{2}\right)\right|_{\mathbb{R}^{n}} \leq \sup _{r \in[0, \sigma]}\left|u_{\mu_{j}, v_{j}}^{\prime \prime}(r)\right|_{\mathbb{R}^{n}}\left|r_{1}-r_{2}\right| \leq \Theta\left|r_{1}-r_{2}\right|, \quad j=1,2, \ldots,
\end{aligned}
$$

for $r_{1}, r_{2} \in[0, \sigma]$. Recalling formulas (3.11) and (3.20), we also obtain

$$
\begin{aligned}
& \left|v_{\mu_{j}, v_{j}}\left(r_{1}\right)-v_{\mu_{j}, v_{j}}\left(r_{2}\right)\right| \leq \sup _{r \in[0, \sigma]}\left|v_{\mu_{j}, v_{j}}^{\prime}(r)\right|\left|r_{1}-r_{2}\right| \\
& \leq \sup _{r \in[0, \sigma]}\left|-\bar{h}^{\prime}(r)+K\left(\bar{h}(r)+v_{j}(r), \bar{f}(r)+u_{\mu_{j}, v_{j}}(r), \bar{f}^{\prime}(r)+u_{\mu_{j}, v_{j}}^{\prime}(r), \phi^{\prime}(r)\right)\right|\left|r_{1}-r_{2}\right| \\
& \leq \theta \sup _{r \in[0, \sigma]}\left(\left|v_{j}(r)\right|+\left|u_{\mu_{j}, v_{j}}(r)\right|_{\mathbb{R}^{n}}+\left|u_{\mu_{j}, v_{j}}^{\prime}(r)\right| \mathbb{R}^{n}\right)\left|r_{1}-r_{2}\right| \\
& \quad \leq \theta\left(\sigma^{2} \Sigma+\sigma \Theta\right)\left|r_{1}-r_{2}\right|, \quad j=1,2, \ldots, r_{1}, r_{2} \in[0, \sigma] .
\end{aligned}
$$


It follows that the sequences $\left(u_{\mu_{j}, v_{j}}\right)_{j=1}^{\infty},\left(u_{\mu_{j}, v_{j}}^{\prime}\right)_{j=1}^{\infty}$, and $\left(v_{\mu_{j}, v_{j}}\right)_{j=1}^{\infty}$ are equicontinuous. Furthermore, because $\mathcal{C} B\left(L_{0}\right)$ is a subset of $B\left(L_{0}\right)$, they are uniformly bounded. These facts, along with the Arzelà-Ascoli theorem, imply that $\left(\left(u_{\mu_{j}, v_{j}}, v_{\mu_{j}, v_{j}}\right)\right)_{j=1}^{\infty}$ must have a convergent subsequence.

According to the lemmas above, the map $\mathcal{C}: B\left(L_{0}\right) \rightarrow \mathcal{B}$ is continuous, and its image is a precompact subset of $B\left(L_{0}\right)$. Consequently, the Schauder theorem (see, e.g. [20, Chapter XII,Corollary 0.1]) allows us to conclude that there exists a pair $(u, v) \in B\left(L_{0}\right)$ satisfying the equality $\mathcal{C}(u, v)=(u, v)$. It is easy to understand that $u$ and $v$ obey (3.12) and (3.13). A simple bootstrapping argument demonstrates that $u$ and $v$ are smooth. We define $f=\bar{f}+u$ and $h=\bar{h}+v$. Clearly, these functions take values in $(0, \infty)^{n}$ and $(0, \infty)$, respectively, and solve (3.5) and (3.7) under the conditions (3.8) and (3.9). Thus, Lemma 3.3 is established.

\subsection{Proof of Theorems 2.5 and 2.6}

Given $\tau \in[0, \sigma]$ and $\kappa>0$, set

$$
J_{\kappa}^{\tau}=(\tau-\kappa, \tau+\kappa) \cap[0, \sigma], \quad \mathcal{X}_{\kappa}^{\tau}=J_{\kappa}^{\tau} \times \mathcal{G} / \mathcal{H} .
$$

Obviously, $\mathcal{X}_{\kappa}^{\tau}$ is a neighbourhood of $\Gamma^{\tau}$ in $M$. Assume that $G^{\tau}$ is a Riemannian metric on $M$ and $G_{\Gamma^{\tau}}^{\tau}$ is the metric on $\Gamma^{\tau}$ induced by $G^{\tau}$. Let $\operatorname{II}\left(G^{\tau}\right)$ be the second fundamental form of $\Gamma^{\tau}$ in $M$ with respect to $G^{\tau}$ and to the unit normal whose scalar product with $\left(\frac{\partial}{\partial r}, 0\right)$ is less than 0 .

For each $\tau \in[0, \sigma]$, consider a symmetric positive definite $\mathcal{G}$-invariant $(0,2)$-tensor field $R^{\tau}$ on $\Gamma^{\tau}$. In order to keep our notation consistent, we assume that $R^{0}$ and $R^{\sigma}$ are the restrictions of $R$ to $\{0\} \times \mathcal{G} / \mathcal{H}$ and $\{\sigma\} \times \mathcal{G} / \mathcal{H}$. It is evident that $R^{\tau}$ is fully determined by how it acts on $\mathfrak{p}$. There exist numbers $a_{\tau, 1}, \ldots, a_{\tau, n}>0$ satisfying

$$
R^{\tau}(X, Y)=a_{\tau, 1}^{2} Q\left(\operatorname{pr}_{\mathfrak{p}_{1}} X, \operatorname{pr}_{\mathfrak{p}_{1}} Y\right)+\cdots+a_{\tau, n}^{2} Q\left(\operatorname{pr}_{\mathfrak{p}_{n}} X, \operatorname{pr}_{\mathfrak{p}_{n}} Y\right), \quad X, Y \in \mathfrak{p} .
$$

Let us also fix, for every $\tau \in[0, \sigma]$, a symmetric $\mathcal{G}$-invariant tensor field $S^{\tau}$ on $\Gamma^{\tau}$. There are $\delta_{\tau, 1}, \ldots, \delta_{\tau, n} \in \mathbb{R}$ such that

$$
S^{\tau}(X, Y)=\delta_{\tau, 1} Q\left(\operatorname{pr}_{\mathfrak{p}_{1}} X, \operatorname{pr}_{\mathfrak{p}_{1}} Y\right)+\cdots+\delta_{\tau, n} Q\left(\operatorname{pr}_{\mathfrak{p}_{n}} X, \operatorname{pr}_{\mathfrak{p}_{n}} Y\right), \quad X, Y \in \mathfrak{p} .
$$

Proposition 3.9, which we are about to state, underlies Theorems 2.5 and 2.6. The author's paper [24] contains similar results, though established in a different setting. Another closely related theorem was obtained in [3]. The methods we use in the present paper are different from those of [24], as we explain in Remark 3.10 below.

Proposition 3.9 Suppose that $\tau \in[0, \sigma]$. The following two statements are equivalent:

1. For some $\kappa>0$, there exists a $\mathcal{G}$-invariant Riemannian metric $G^{\tau}$ on $M$ such that $\operatorname{Ric}\left(G^{\tau}\right)=T$ on $\mathcal{X}_{\kappa}^{\tau}, G_{\Gamma^{\tau}}^{\tau}=R^{\tau}$, and $\operatorname{II}\left(G^{\tau}\right)=S^{\tau}$.

2. The inequality

$$
\sum_{k=1}^{n} d_{k}\left(\frac{\beta_{k}}{2 a_{\tau, k}^{2}}+\sum_{l, m=1}^{n} \gamma_{k, l}^{m} \frac{a_{\tau, k}^{4}-2 a_{\tau, l}^{4}}{4 a_{\tau, k}^{2} a_{\tau, l}^{2} a_{\tau, m}^{2}}-\sum_{l=1}^{n} d_{l} \frac{\delta_{\tau, k} \delta_{\tau, l}}{a_{\tau, k}^{2} a_{\tau, l}^{2}}+\frac{\delta_{\tau, k}^{2}}{a_{\tau, k}^{4}}-\frac{1}{a_{\tau, k}^{2}} \phi_{k}(\tau)\right)<0
$$

is satisfied. 
If these statements hold and $\check{G}^{\tau}$ is a $\mathcal{G}$-invariant metric on $M$ such that $\operatorname{Ric}\left(\check{G}^{\tau}\right)=T$ on $\mathcal{X}_{\kappa}^{\tau}$, $\check{G}_{\Gamma}^{\tau} \tau=R^{\tau}$, and $\operatorname{II}\left(\check{G}^{\tau}\right)=S^{\tau}$, then $\check{G}^{\tau}$ must coincide with $G^{\tau}$ on $\mathcal{X}_{\kappa}^{\tau}$.

Proof Suppose that there exist $\kappa>0$ and a $\mathcal{G}$-invariant Riemannian metric $G^{\tau}$ on $M$ such that $\operatorname{Ric}\left(G^{\tau}\right)=T$ on $\mathcal{X}_{\kappa}^{\tau}, G_{\Gamma^{\tau}}^{\tau}=R^{\tau}$, and $\operatorname{II}\left(G^{\tau}\right)=S^{\tau}$. Employing Lemma 3.1 and the fact that $T$ is positive definite, one can show that $G^{\tau}$ satisfies the formula

$$
G^{\tau}=h_{\tau}^{2}(r) \mathrm{d} r \otimes \mathrm{d} r+G_{r}^{\tau}, \quad r \in J_{\kappa}^{\tau} .
$$

Here, $h_{\tau}$ is a smooth function acting from $J_{\kappa}^{\tau}$ to $(0, \infty)$. The tensor field $G_{r}^{\tau}$ is a $\mathcal{G}$-invariant Riemannian metric on $\mathcal{G} / \mathcal{H}$. It is clear that

$$
G_{r}^{\tau}(X, Y)=f_{\tau, 1}^{2}(r) Q\left(\operatorname{pr}_{\mathfrak{p}_{1}} X, \operatorname{pr}_{\mathfrak{p}_{1}} Y\right)+\cdots+f_{\tau, n}^{2}(r) Q\left(\operatorname{pr}_{\mathfrak{p}_{n}} X, \operatorname{pr}_{\mathfrak{p}_{n}} Y\right), \quad X, Y \in \mathfrak{p},
$$

for some smooth functions $f_{\tau, 1}, \ldots, f_{\tau, n}$ from $J_{\kappa}^{\tau}$ to $(0, \infty)$. The equality $\operatorname{Ric}\left(G^{\tau}\right)=T$ and Lemma 3.1 imply

$$
H_{1}\left(f_{\tau}(r), f_{\tau}^{\prime}(r)\right)=h_{\tau}^{2}(r) H_{2}\left(f_{\tau}(r), \phi(r)\right), \quad r \in J_{\kappa}^{\tau} .
$$

The notation $f_{\tau}$ here stands for $\left(f_{\tau, 1}, \ldots, f_{\tau, n}\right)$. Because $G_{\Gamma \tau}^{\tau}=R^{\tau}$ and $\operatorname{II}\left(G^{\tau}\right)=S^{\tau}$, we also have

$$
f_{\tau}(\tau)=a_{\tau}, \quad f_{\tau}^{\prime}(\tau)=-h_{\tau}(\tau) \delta_{\tau}^{a}
$$

where $a_{\tau}=\left(a_{\tau, 1}, \ldots, a_{\tau, n}\right)$ and $\delta_{\tau}^{a}=\left(\frac{\delta_{\tau, 1}}{a_{\tau, 1}}, \ldots, \frac{\delta_{\tau, n}}{a_{\tau, n}}\right)$. Keeping these two formulas in mind and using (3.25), we easily calculate that the quantity in the left-hand side of (3.22) is equal to $-\frac{1}{h_{\tau}^{2}(\tau)}$. This quantity must, therefore, be negative.

Assume now that (3.22) holds. Let us prove the existence of $\kappa>0$ and a metric $G^{\tau}$ on $M$ such that $\operatorname{Ric}\left(G^{\tau}\right)=T$ on $\mathcal{X}_{\kappa}^{\tau}, G_{\Gamma^{\tau}}^{\tau}=R^{\tau}$, and $\operatorname{II}\left(G^{\tau}\right)=S^{\tau}$. Consider the system of ordinary differential equations

$$
\begin{aligned}
& f_{\tau}^{\prime \prime}(r)=\bar{F}\left(h_{\tau}(r), f_{\tau}(r), f_{\tau}^{\prime}(r), \phi(r), \phi^{\prime}(r)\right), \\
& h_{\tau}^{\prime}(r)=K\left(h_{\tau}(r), f_{\tau}(r), f_{\tau}^{\prime}(r), \phi^{\prime}(r)\right),
\end{aligned}
$$

for the unknown functions $f_{\tau}$ and $h_{\tau}$. We supplement this system with the conditions

$$
\begin{aligned}
& f_{\tau}(\tau)=a_{\tau}, \\
& f_{\tau}^{\prime}(\tau)=-\left(H_{2}\left(a_{\tau}, \phi(\tau)\right)+\left(1-H_{1}\left(a_{\tau}, \delta_{\tau}^{a}\right)\right)\right)^{-\frac{1}{2}} \delta_{\tau}^{a}, \\
& h_{\tau}(\tau)=\left(H_{2}\left(a_{\tau}, \phi(\tau)\right)+\left(1-H_{1}\left(a_{\tau}, \delta_{\tau}^{a}\right)\right)\right)^{-\frac{1}{2}} .
\end{aligned}
$$

Note that, thanks to (3.22), the right-hand sides of the last two formulas are well defined. The standard theory of ordinary differential equations tells us that problem (3.26)-(3.27) has a solution. To be more precise, for some number $\kappa>0$, there exist smooth functions $f_{\tau}: J_{\kappa}^{\tau} \rightarrow(0, \infty)^{n}$ and $h_{\tau}: J_{\kappa}^{\tau} \rightarrow(0, \infty)$ solving (3.26) on $J_{\kappa}^{\tau}$ and satisfying (3.27). With these functions at hand, we define a $\mathcal{G}$-invariant Riemannian metric $G^{\tau}$ on $\mathcal{X}_{\kappa}^{\tau}$ by formulas (3.23) and (3.24). We extend it to all of $M$ arbitrarily. It follows from (3.26) that

$$
\operatorname{Ric}\left(G^{\tau}\right)=\hat{\sigma}(r) \mathrm{d} r \otimes \mathrm{d} r+T_{r}, \quad r \in J_{\kappa}^{\tau},
$$

for some $\hat{\sigma}: J_{\kappa}^{\tau} \rightarrow \mathbb{R}$. Employing Lemma 3.2 and arguing as in Sect. 3.2, one demonstrates that $\hat{\sigma}$ must be identically equal to 1 on $J_{\kappa}^{\tau}$. This means $\operatorname{Ric}\left(G^{\tau}\right)=T$ on $\mathcal{X}_{\kappa}^{\tau}$. Conditions (3.27) imply that $G_{\Gamma^{\tau}}^{\tau}=R^{\tau}$ and $\operatorname{II}\left(G^{\tau}\right)=S^{\tau}$. 
Suppose now that statements 1 and 2 in Proposition 3.9 hold true. We may assume that the metric $G^{\tau}$ satisfies (3.23) and (3.24). Then, the functions $f_{\tau}$ and $h_{\tau}$ solve (3.26)-(3.27) on $J_{\kappa}^{\tau}$. Consider a $\mathcal{G}$-invariant Riemannian metric $\check{G}^{\tau}$ on $M$ such that $\operatorname{Ric}\left(\check{G}^{\tau}\right)=T$ on $\mathcal{X}_{\kappa}^{\tau}$, $\check{G}_{\Gamma^{\tau}}^{\tau}=R^{\tau}$, and $\operatorname{II}\left(\check{G}^{\tau}\right)=S^{\tau}$. Our objective is to show that $\check{G}^{\tau}$ coincides with $G^{\tau}$ on $\mathcal{X}_{\kappa}^{\tau}$. By analogy with (3.23), we write

$$
\check{G}^{\tau}=\check{h}_{\tau}^{2}(r) \mathrm{d} r \otimes \mathrm{d} r+\check{G}_{r}^{\tau}, \quad r \in J_{\kappa}^{\tau} .
$$

In the right-hand side, $\breve{h}_{\tau}: J_{\kappa}^{\tau} \rightarrow(0, \infty)$ is a smooth function. The tensor field $\check{G}_{r}^{\tau}$ is a $\mathcal{G}$-invariant Riemannian metric on $\mathcal{G} / \mathcal{H}$. There are smooth functions $\check{f}_{\tau, 1}, \ldots, \check{f}_{\tau, n}$ from $J_{\kappa}^{\tau}$ to $(0, \infty)$ such that

$$
\check{G}_{r}^{\tau}(X, Y)=\check{f}_{\tau, 1}^{2}(r) Q\left(\operatorname{pr}_{\mathfrak{p}_{1}} X, \operatorname{pr}_{\mathfrak{p}_{1}} Y\right)+\cdots+\check{f}_{\tau, n}^{2}(r) Q\left(\operatorname{pr}_{\mathfrak{p}_{n}} X, \operatorname{pr}_{\mathfrak{p}_{n}} Y\right), \quad X, Y \in \mathfrak{p} .
$$

It will be convenient for us to denote $\check{f}_{\tau}=\left(\check{f}_{\tau, 1}, \ldots, \check{f}_{\tau, n}\right)$. Because $\operatorname{Ric}\left(\check{G}^{\tau}\right)=T, \check{G}_{\Gamma}^{\tau}=$ $R^{\tau}$, and II $\left(\check{G}^{\tau}\right)=S^{\tau}$, formulas (3.26)-(3.27) would still hold on $J_{\kappa}^{\tau}$ if we substituted $\check{f}_{\tau}, \check{f}_{\tau}^{\prime}$, $\check{f}_{\tau}^{\prime \prime}, \check{h}_{\tau}$, and $\check{h}_{\tau}^{\prime}$ in them for $f_{\tau}, f_{\tau}^{\prime}, f_{\tau}^{\prime \prime}, h_{\tau}$, and $h_{\tau}^{\prime}$. The standard theory of ordinary differential equations then implies that $\check{f}_{\tau}=f_{\tau}$ and $\check{h}_{\tau}=h_{\tau}$ on $J_{\kappa}^{\tau}$. Consequently, $\check{G}^{\tau}$ coincides with $G^{\tau}$ on $\mathcal{X}_{\kappa}^{\tau}$. Thus, the proof is complete.

Remark 3.10 One may establish Proposition 3.9 by adapting the methods employed in the paper [24]. The main idea behind those methods is to modify the right-hand side of (1.1) by a $G$-dependent diffeomorphism making the equation more easily solvable. Such an approach relies on the work of DeTurck (see [7, Chapter 5] for an overview) and is similar in spirit to the DeTurck trick for the Ricci flow. Conversely, it seems possible to prove the existence and uniqueness results in [24] with the techniques employed above.

Remark 3.11 Suppose that we are in the situation described in Remark 2.8. Thus, $\mathcal{G} / \mathcal{H}$ is an abelian Lie group, and Hypothesis 2.1 fails to hold. In this case, statement 2 of Proposition 3.9 implies statement 1 , but establishing the converse implication may be problematic. Roughly speaking, this is because, when Hypothesis 2.1 does not hold, the metric $G^{\tau}$ need not be diagonal with respect to (2.1). For the same reason, proving the assertion about $\breve{G}^{\tau}$ may be troublesome with our methods.

Remark 3.12 Statement 1 of Proposition 3.9 is equivalent to statement 2 even if $T$ is not positive definite. Yet, our methods do not yield the assertion about $\check{G}^{\tau}$ in this case. Recall that if $T$ is not positive definite, we need to assume the existence of a diffeomorphism $\Psi$ satisfying (2.2), (2.3) and (2.4).

Theorems 2.5 and 2.6 follow from Proposition 3.9 by choosing $R^{\tau}$ and $S^{\tau}$ in such a way that

$$
\begin{aligned}
R^{\tau}(X, Y)= & \left.\frac{\sigma-\tau}{\sigma} a_{1}+\frac{\tau}{\sigma} b_{1}\right) Q\left(\operatorname{pr}_{\mathfrak{p}_{1}} X, \operatorname{pr}_{\mathfrak{p}_{1}} Y\right) \\
& +\cdots+\left(\frac{1-\tau}{\sigma} a_{n}+\frac{\tau}{\sigma} b_{n}\right) Q\left(\operatorname{pr}_{\mathfrak{p}_{n}} X, \operatorname{pr}_{\mathfrak{p}_{n}} Y\right), \\
S^{\tau}(X, Y) & =\beta Q(X, Y), \quad X, Y \in \mathfrak{p},
\end{aligned}
$$

for a sufficiently large $\beta>0$. 


\section{References}

1. Anderson, M.T.: On boundary value problems for Einstein metrics. Geom. Topol. 12, 2009-2045 (2008)

2. Anderson, M.T.: Boundary value problems for metrics on 3-manifolds. In: Dai, X., Rong, X. (eds.) Metric and Differential Geometry, in honor of J. Cheeger, pp. 3-17. Birkhäuser Verlag, Basel (2012)

3. Anderson, M.T., Herzlich, M.: Unique continuation results for Ricci curvature and applications. J. Geom. Phys. 58, 179-207 (2008) ; erratum in J. Geom. Phys. 60, 1062-1067 (2010)

4. Aubin, T.: Some Nonlinear Problems in Riemannian Geometry. Springer, Berlin (1998)

5. Bailesteanu, M., Cao, X., Pulemotov, A.: Gradient estimates for the heat equation under the Ricci flow. J. Funct. Anal. 258, 3517-3542 (2010)

6. Bérard Bergery, L.: Sur de nouvelles varietes riemanniennes d'Einstein, Institut Élie Cartan 6, pp. 1-60 (1982)

7. Besse, A.: Einstein Manifolds. Springer, Berlin (1987)

8. Böhm, C.: Inhomogeneous Einstein metrics on low-dimensional spheres and other low-dimensional spaces. Invent. Math. 134, 145-176 (1998)

9. Cao, J., DeTurck, D.M.: The Ricci curvature equation with rotational symmetry. Am. J. Math. 116, 219-241 (1994)

10. Cortissoz, J.C.: Three-manifolds of positive curvature and convex weakly umbilic boundary. Geom. Dedicata 138, 83-98 (2009)

11. Dancer, A.S., Wang, M.Y.: Integrable cases of the Einstein equations. Commun. Math. Phys. 208, 225-243 (1999)

12. Dancer, A.S., Wang, M.Y.: The cohomogeneity one Einstein equations from the Hamiltonian viewpoint. J. Reine Angew. Math. 524, 97-128 (2000)

13. Dancer, A.S., Wang, M.Y.: On Ricci solitons of cohomogeneity one. Ann. Glob. Anal. Geom. 39, 259-292 (2011)

14. DeBlois, J., Knopf, D., Young, A.: Cross curvature flow on a negatively curved solid torus. Algebr. Geom. Topol. 10, 343-372 (2010)

15. Delanoë, P.: Local solvability of elliptic, and curvature, equations on compact manifolds. J. Reine Angew. Math. 558, 23-45 (2003)

16. Delay, E.: Studies of some curvature operators in a neighborhood of an asymptotically hyperbolic Einstein manifold. Adv. Math. 168, 213-224 (2002)

17. Gianniotis, P.: The Ricci flow on manifolds with boundary, arXiv:1210.0813 [math.DG]

18. Grove, K., Ziller, W.: Cohomogeneity one manifolds with positive Ricci curvature. Invent. Math. 149, 619-646 (2002)

19. Hamilton, R.S.: The Ricci curvature equation. In: Chern, S.-S. (ed.) Seminar on Nonlinear Partial Differential Equations, pp. 47-72. Springer, New York (1984)

20. Hartman, P.: Ordinary Differential Equations. Wiley, New York (1964)

21. Hoelscher, C.A.: Classification of cohomogeneity one manifolds in low dimensions. Pac. J. Math. 246, 129-185 (2010)

22. Pina, R., Tenenblat, K.: On solutions of the Ricci curvature equation and the Einstein equation. Isr. J. Math. 171, 61-76 (2009)

23. Pulemotov, A.: Quasilinear parabolic equations and the Ricci flow on manifolds with boundary. J. Reine Angew. Math. 683, 97-118 (2013)

24. Pulemotov, A.: Metrics with prescribed Ricci curvature near the boundary of a manifold. Math. Ann. 357, 969-986 (2013)

25. Pulemotov A.: The Ricci flow on domains in cohomogeneity one manifolds, submitted, arXiv:1410.7505 [math.AP]

26. Pulemotov A.: Metrics with prescribed Ricci curvature on homogeneous spaces, submitted, arXiv: 1504.01498 [math.DG]

27. Rubinstein, Y.A.: Some discretizations of geometric evolution equations and the Ricci iteration on the space of Kähler metrics. Adv. Math. 218, 1526-1565 (2008)

28. Rubinstein, Y.A.: Some discretizations of geometric evolution equations and the Ricci iteration on the space of Kähler metrics, Ph.D. thesis, Massachusetts Institute of Technology (2008)

29. Shen, Y.: On Ricci deformation of a Riemannian metric on manifold with boundary. Pac. J. Math. 173, 203-221 (1996) 